\title{
HPV testing in routine cervical screening: cross sectional data from the ARTISTIC trial
}

\author{
HC Kitchener, ${ }^{*}$, M Almonte ${ }^{2}$, P Wheeler ${ }^{3}$, M Desai ${ }^{4}$, C Gilham ${ }^{5}$, A Bailey ${ }^{6}$, A Sargent ${ }^{7}$ and J Peto $^{8,9}$ on behalf of \\ the ARTISTIC Trial Study Group ${ }^{10}$
}

'Division of Human Development, University of Manchester, Hathersage Road, Manchester MI 3 OJH, UK; ${ }^{2}$ Non-Communicable Disease Epidemiology Unit, London School of Hygiene and Tropical Medicine, Keppel Street, London WCIE 7HT, UK; ${ }^{3}$ Division of Human Development, University of Manchester, Hathersage Road, Manchester M 33 OJH, UK; ${ }^{4}$ Department of Cytology, Central Manchester and Manchester Children's University Hospitals NHS Trust, Oxford Road, Manchester M 33 9WL, UK; ${ }^{5}$ Cancer Research UK Epidemiology and Genetics Unit, Institute of Cancer Research, Sutton, Surrey SM2 5NG, UK; ${ }^{6}$ Department of Virology, Central Manchester and Manchester Children's University Hospitals NHS Trust, Oxford Road, Manchester M I 3 9WL, UK; ${ }^{7}$ Division of Human Development, University of Manchester, Hathersage Road, Manchester MI 3 OJH, UK; ${ }^{8}$ Non-Communicable Disease Epidemiology Unit, London School of Hygiene and Tropical Medicine, Keppel Street, London WCIE 7HT, UK; ${ }^{9}$ Cancer Research UK Epidemiology and Genetics Unit, Institute of Cancer Research, Sutton, Surrey SM2 5NG, UK

To evaluate the effectiveness of human papillomavirus (HPV) testing in primary cervical screening. This was a cross-sectional study from the recruitment phase of a prospective randomised trial. Women were screened for HPV in addition to routine cervical cytology testing. Greater Manchester, attendees at routine NHS Cervical Screening Programme. In all, 245I0 women aged 20-64 screened with liquid-based cytology (LBC) and HPV testing at entry. HPV testing in primary cervical screening. Type-specific HPV prevalence rates are presented in relation to age as well as cytological and histological findings at entry. In all, 24510 women had adequate cytology and HPV results. Cytology results at entry were: $87 \%$ normal, $11 \%$ borderline or mild, $1.1 \%$ moderate and $0.6 \%$ severe dyskaryosis or worse. Prevalence of HPV decreased sharply with age, from $40 \%$ at age $20-24$ to $12 \%$ at $35-39$ and $7 \%$ or less above age 50 . It increased with cytological grade, from $10 \%$ of normal cytology and $31 \%$ of borderline to $70 \%$ mild, $86 \%$ moderate, and $96 \%$ of severe dyskaryosis or worse. HPV 16 or HPV 18 accounted for $64 \%$ of infections in women with severe or worse cytology, and one or both were found in $61 \%$ of women with severe dyskaryosis but in only $2.2 \%$ of those with normal cytology. The majority of young women in Greater Manchester have been infected with a high-risk HPV by the age of 30. HPV testing is practicable as a primary routine screening test, but in women aged under 30 years, this would lead to a substantial increase in retesting and referral rates. HPV 16 and HPV 18 are more predictive of underlying disease, but other HPV types account for $30 \%$ of high-grade disease.

British Journal of Cancer (2006) 95, 56-61. doi: 10.1038/sj.bjc.66032I0 www.bjcancer.com

Published online 13 June 2006

(c) 2006 Cancer Research UK

Keywords: cervical screening; HPV detection; typing; cytology

Cervical cancer is the second most common cancer among women worldwide, and ranks first in many developing countries (Parkin, 2004). Human papillomaviruses (HPV), most frequently HPV 16, are the primary cause of cervical carcinogenesis (IARC, 1995). Over $100 \mathrm{HPV}$ types have now been described, including about 20 'high-risk' types that are associated with cervical cancer (Clifford et al, 2005). The overall prevalence of HPV in cervical cancers in a large international study was over $99 \%$, implying the highest attributable fraction ever identified for a specific cause of a major human cancer (Walboomers et al, 1999). In many developed countries, particularly the UK, systematic cervical screening based on cytology has been responsible for a significant fall in the incidence and death rate from cervical cancer (Peto et al, 2004). The NHS Cervical Screening Programme in England now offers screening 3 yearly between ages 25 and 49 and 5 yearly between 50

* Correspondence: Professor HC Kitchener,

E-mail: henry.kitchenen@cmmc.nhs.uk

${ }^{10}$ See Appendix.

Received 17 January 2006; revised 8 May 2006; accepted II May 2006; published online 13 June 2006 and 64 years, and liquid-based cytology (LBC) is currently being implemented. Nonrandomised studies have shown that HPV DNA testing is more sensitive than cytology for detecting CIN, and the International Agency for Research on Cancer (IARC) recently concluded that testing for HPV as a primary screening modality could reduce cancer incidence and mortality (IARC, 2004).

In order to evaluate the effectiveness of HPV testing in primary cervical screening, the Trial, A Randomised Trial in Screening to Improve Cytology (ARTISTIC) Trial is being conducted within the routine NHS Cervical Screening Programme in Greater Manchester. This randomised trial will compare outcomes in women whose HPV test result is concealed with those in whom it is revealed and acted upon. In this paper, we report prevalence rates of HPV 16, HPV 18 and other high-risk HPVs in relation to age, cytology and histology at entry to the trial.

\section{METHODS}

Between July 2001 and October 2003, women aged 20-64 years attending for routine cervical screening in four health authorities in the Manchester area (Manchester, Salford \& Trafford, Stockport 
and Wigan \& Leigh) were invited to participate. The trial, which was approved by the North West Multicentre Research Ethics Committee, was explained in a letter enclosed with the invitation to attend for a routine smear. Women willing to participate were asked to sign informed consent before having a smear collected for LBC and HPV testing. This is a population-based study and is therefore representative of women in Greater Manchester, a heavily populated British conurbation.

The cervical sample was collected using the broom-like device of the Thin-Prep ${ }^{\mathrm{TM}}$ (Cytyc) kit and rinsed into a vial containing PreservCyt ${ }^{\circledR}$ transport medium. The sample and consent form were sent to the Manchester Cytology Centre laboratory in Manchester Royal Infirmary if they were collected in Manchester, Salford \& Trafford, or Wigan \& Leigh, and to Stepping Hill Hospital cytology laboratory if they were from the Stockport area. Samples accompanied by a consent form were flagged on receipt on the laboratory database. Consent forms were checked and forwarded to the ARTISTIC trial office, which sent a letter notifying participants of their random allocation (HPV result revealed or concealed).

\section{Design of the trial}

The data reported in this study are derived from the cytology and HPV testing performed during the recruitment phase. Figure 1 shows the design of the trial which will be completed in 2006/7. Women were randomised in a 3:1 ratio to the revealed arm or the concealed arm. In both arms the cervical sample was processed for cytology and then sent to the virology laboratory. HPV testing and cytology were thus conducted independently. Women with inadequate cytology at first visit were resampled. All women are being recalled for a second round of screening by LBC and HPV testing at 36 months.

\section{Concealed arm}

All HPV results at entry and subsequently are concealed from the women and from clinical staff including the HPV results at 36 months. All women in the concealed arm, and those in the revealed arm who remained HPV-negative, were referred to colposcopy according to national guidelines at that time, that is (i) after three consecutive inadequate samples; (ii) after three consecutive borderline smears; (iii) after an initial borderline or mildly dyskaryotic smear followed by another showing mild dyskaryosis or worse; or (iv) following a single smear showing moderate dyskaryosis or worse.

\section{Revealed arm}

Women in the revealed arm with normal cytology at entry who tested HPV positive were invited for a second HPV test 12 months after entry. If this second test was also positive, they were invited to choose between immediate colposcopy or a repeat HPV test after a further 12 months. If this third test was again positive, women were referred to colposcopy.

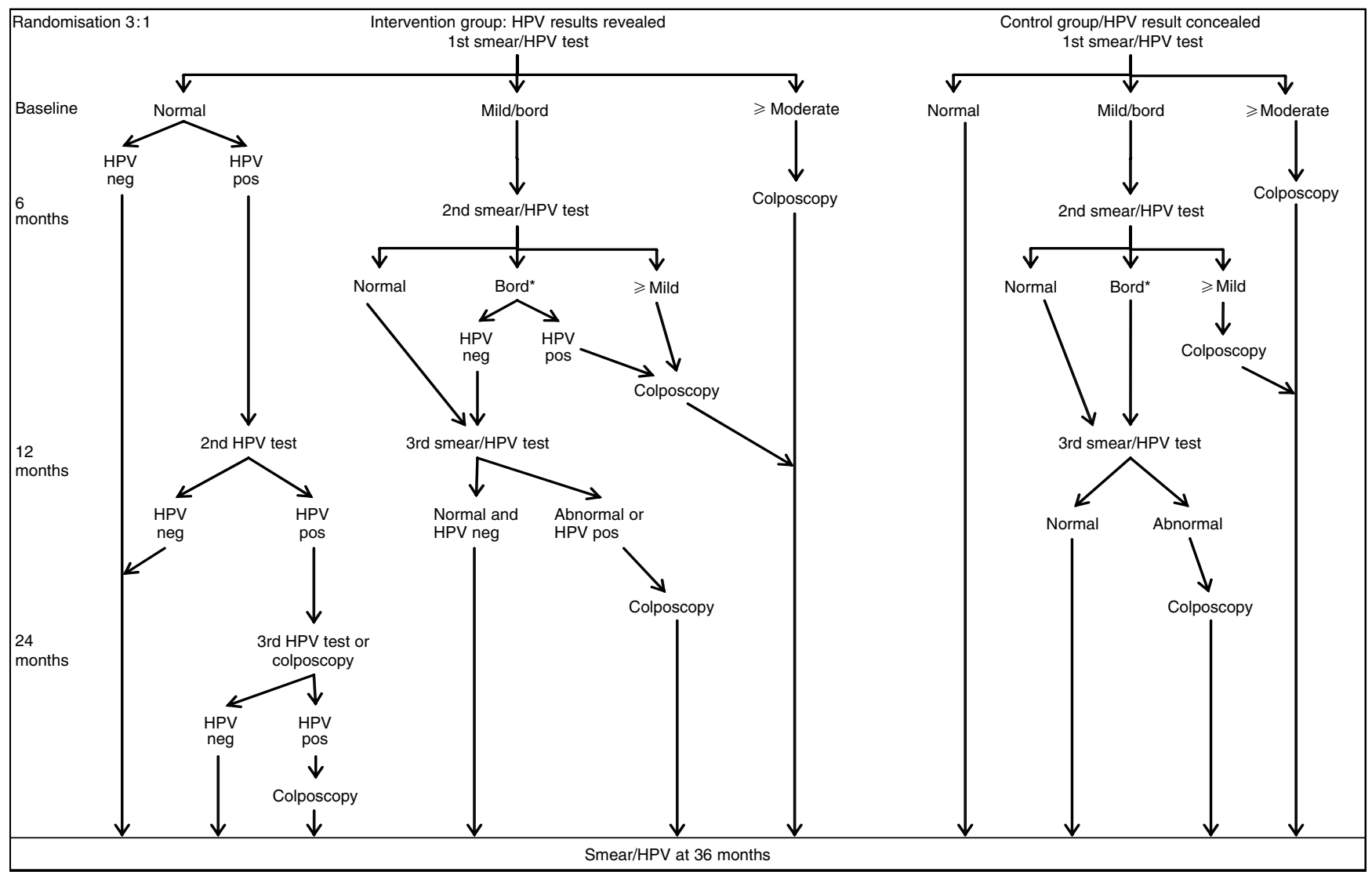

* Referral to colposcopy vary depending on cytology history, some women could be referred to colposcopy only after three consecutive borderline smears or after a mild followed by a borderline smear.

Figure I ARTISTIC Trial protocol for the management of women with normal and abnormal cytology and HPV-positive and -negative tests, in the revealed arm 


\section{Outcome measures}

Primary outcome measures will include the proportion of additional CIN3 lesions diagnosed in the revealed arm in cytologically normal women who were HPV positive at entry and any resulting reduction in their CIN3 detection rate at the 3 year second screening round (CIN3 cases diagnosed in women who are cytologically normal at 3 years will be ignored in this concealed/ revealed comparison). Other relevant outcomes are (a) the sensitivity and specificity of persistently detectable HPV for diagnosis of CIN3 or cancer; (b) the cost effectiveness of HPV testing; (c) the psychological effects of HPV testing; (d) attendance rates among HPV positive women when recalled after 1 year for retesting and (e) their preference between immediate colposcopy and further HPV testing if they are still HPV positive. Some HPV positive women fail to return for a second HPV test after 12 months, and the trial will also provide substantial evidence on the effects of retesting HPV positive women after 3 years, which may prove as sensitive but more specific for CIN3 diagnosis compared with retesting after 1 year.

\section{Laboratory procedures}

LBC using ThinPrep ${ }^{\circledR}$ ThinPrep ${ }^{\circledR}$ vials were collected from the participating practices or clinics and sent either to Manchester Cytology Centre or to Stepping Hill Hospital Cytology Laboratory. Vials not accompanied by a consent form were not processed until the consent form was received at the laboratory. Laboratory numbers were allocated to the samples before automatic staining and routine processing in cytology.

Most were processed using the multi sample ThinPrep ${ }^{\circledR} 3000$ processor (Cytyc). The single sample ThinPrep ${ }^{\mathbb{R}} 2000$ (Cytyc) was used if an extra slide needed to be made from a sample because the initial slide appeared unsatisfactory or if only a few samples needed to be processed.

Vials containing the residual sample were sent to the Virology Laboratory in Manchester Royal Infirmary for HPV testing by the Digene Hybrid Capture ${ }^{\circledR} 2$ (hc2) assay. Laboratory numbers were allocated to the samples before automatic staining and routine processing. Procedures are in place to ensure that cross contamination by HPV does not occur in the T3000 processor and swabs are taken to ensure no contamination is present.

\section{HPV testing using Hybrid Capture ${ }^{\circledR} 2$ (hc2)}

Detection of high-risk HPV genotypes by hc2 assay Cervical samples collected into Cytyc LBC medium were tested for high-risk HPV DNA by hc2 according to the manufacturer's instructions. Briefly, after treatment with conversion buffer, single-stranded DNA (ssDNA) was produced by adding denaturing fluid and heating at $65^{\circ} \mathrm{C}$ for $45 \mathrm{~min}$. Following hybridisation of ssDNA to high-risk HPV specific RNA probes the DNA/RNA hybrids were captured onto anti-DNA/RNA antibody coated micro-titre plates prior to detection of the hybrids using an alkaline phosphatase conjugated anti-DNA/RNA antibody in conjunction with a light emitting substrate. Positive results were expressed in relative light units (RLUs) compared with a positive control containing $1 \mathrm{pg} \mathrm{ml}^{-1}$ of HPV DNA. The high-risk HPV types detected by the assay are $16,18,31,33,35,39,45,51,52,56,58,59$ and 68 . We classified samples as HPV positive according to the manufacturer's instructions at the outset of the study, which was to use a positive cut off as $1 \mathrm{RLU} /$ control.

\section{HPV genotyping (Roche reverse line blot assay)}

Genotyping of hc2 positive samples was carried out using the prototype reverse line blot assay supplied by Roche.
DNA was extracted from $50 \mu \mathrm{l}$ of the pelleted sample stored at $-70^{\circ} \mathrm{C}$ using the automated Roche MagNAPure LC system. Extracted DNA was amplified using the PGMY primer reagents provided by Roche. Biotinylated PCR product was then denatured and captured onto nylon strips coated with HPV type specific oligonucleotides. Immobilised product was visualised using streptavidin-horseradish peroxidase-mediated colour precipitation.

\section{Data management}

Trial participants were flagged on the routine cytology and histology records of both cytology laboratories. In order to avoid the possibility that any histology results have been obtained from the second screening round, we have censored histology records at 2 years. Name, address and date of birth are routinely used by cytology laboratories to match new smears against a woman's previous screening record. A separate database was maintained by the virology laboratory. Relevant results were sent to the trial office in Manchester regularly, where they were matched and appended to participants' records on an Access 2000 database. Stata version 8 statistical software (StataCorp., 2004) was used for all analyses.

\section{RESULTS}

A total of 25020 women were enrolled of whom 397 (1.6\%) had an inadequate first smear at entry including $71(0.3 \%)$ who did not provide an adequate repeat smear. These 71 women were referred for colposcopy according to national guidelines and excluded from further analysis. The cytology results presented thus correspond to the first adequate smear. In all, 85 women had a conventional smear and no HPV test at entry, 137 had samples that were insufficient for HPV testing, 84 were younger than 20 and 133 were older than 64 years of age. There were thus 24510 (18 386 revealed and 6124 concealed) eligible women with satisfactory cytology and HPV results at entry.

\section{Cytology and HPV results}

Table 1 shows that cytology and HPV results at entry did not differ between the randomised arms and are therefore presented together; $87 \%$ of women had normal cytology, $11 \%$ had borderline smears or mild dyskaryosis, $1.1 \%$ had moderate dyskaryosis, and $0.8 \%$ had severe dyskaryosis or worse abnormality. The mean age was 40.2 years in both arms. The prevalence of high-risk HPV detection (Figure 2) declined from $40 \%$ in women aged $20-24$ to $12 \%$ at $35-39$ and $6 \%$ at 55 years or older.

Figure 3 shows the prevalence of HPV infection by age and cytology at entry. HPV prevalence increased with the level of cytological abnormality, proportionally more so at older ages. HPV prevalence rates at age 20-29 years were 55\% (borderline), $87 \%$

Table I Cytology and high-risk HPV results by randomisation arms at entry

\begin{tabular}{|c|c|c|c|c|c|c|}
\hline \multirow[b]{2}{*}{ Cytology } & \multicolumn{3}{|c|}{ Revealed arm } & \multicolumn{3}{|c|}{ Concealed arm } \\
\hline & HPV - & HPV + & Total & HPV - & HPV + & Total \\
\hline Negative & 14367 & 1675 & 16042 (87.3\%) & 4787 & 551 & 5338 (87.2\%) \\
\hline Borderline & 923 & 420 & $1343(7.3 \%)$ & 309 & 137 & 446 (7.3\%) \\
\hline Mild & 196 & 447 & 643 (3.5\%) & 69 & 166 & 235 (3.8\%) \\
\hline Moderate & 34 & 170 & $204(1.1 \%)$ & 4 & 63 & 67 (1.1\%) \\
\hline Severe/worse & 6 & 148 & $154(0.8 \%)$ & 2 & 36 & $38(0.6 \%)$ \\
\hline Total & 15526 & 2860 & $18386(100 \%)$ & 5171 & 953 & 6124 (100\%) \\
\hline
\end{tabular}




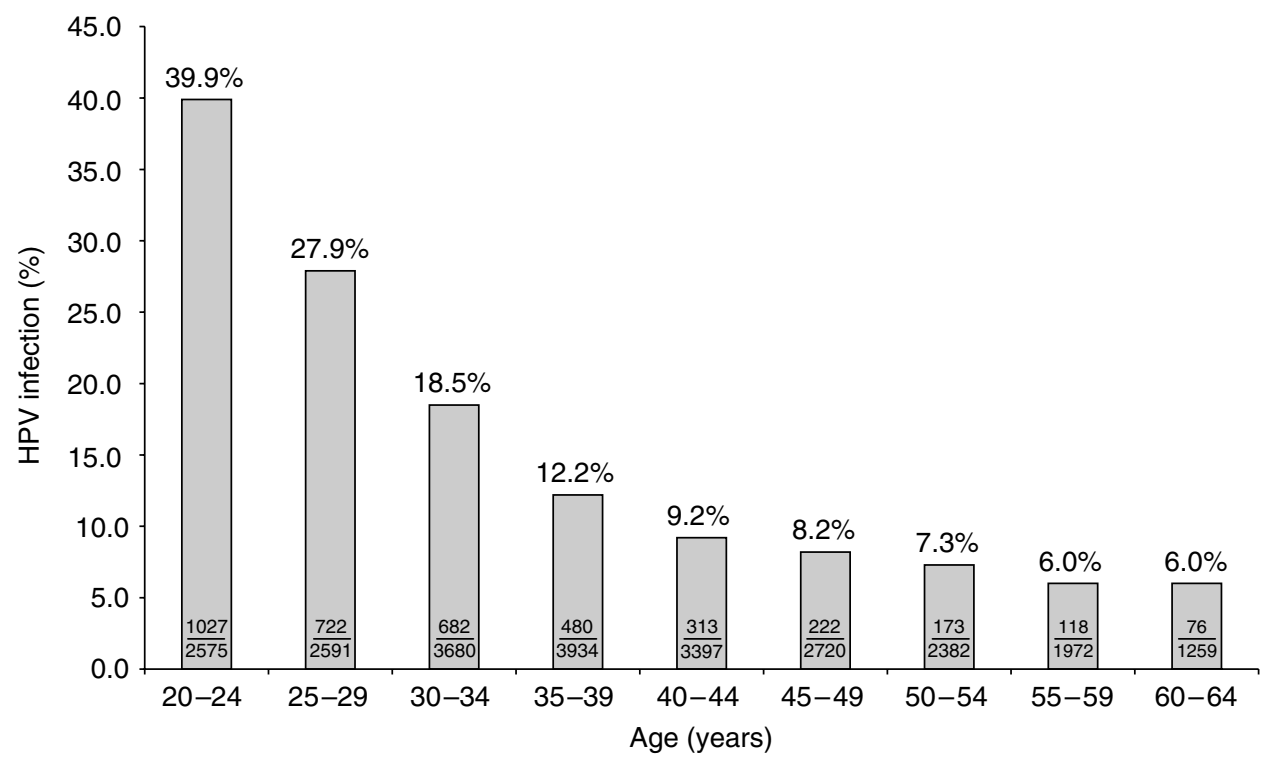

Figure 2 Prevalence of high-risk HPV (HR HPV) by Hybrid Capture 2 (HC2) according to age quinquennia.

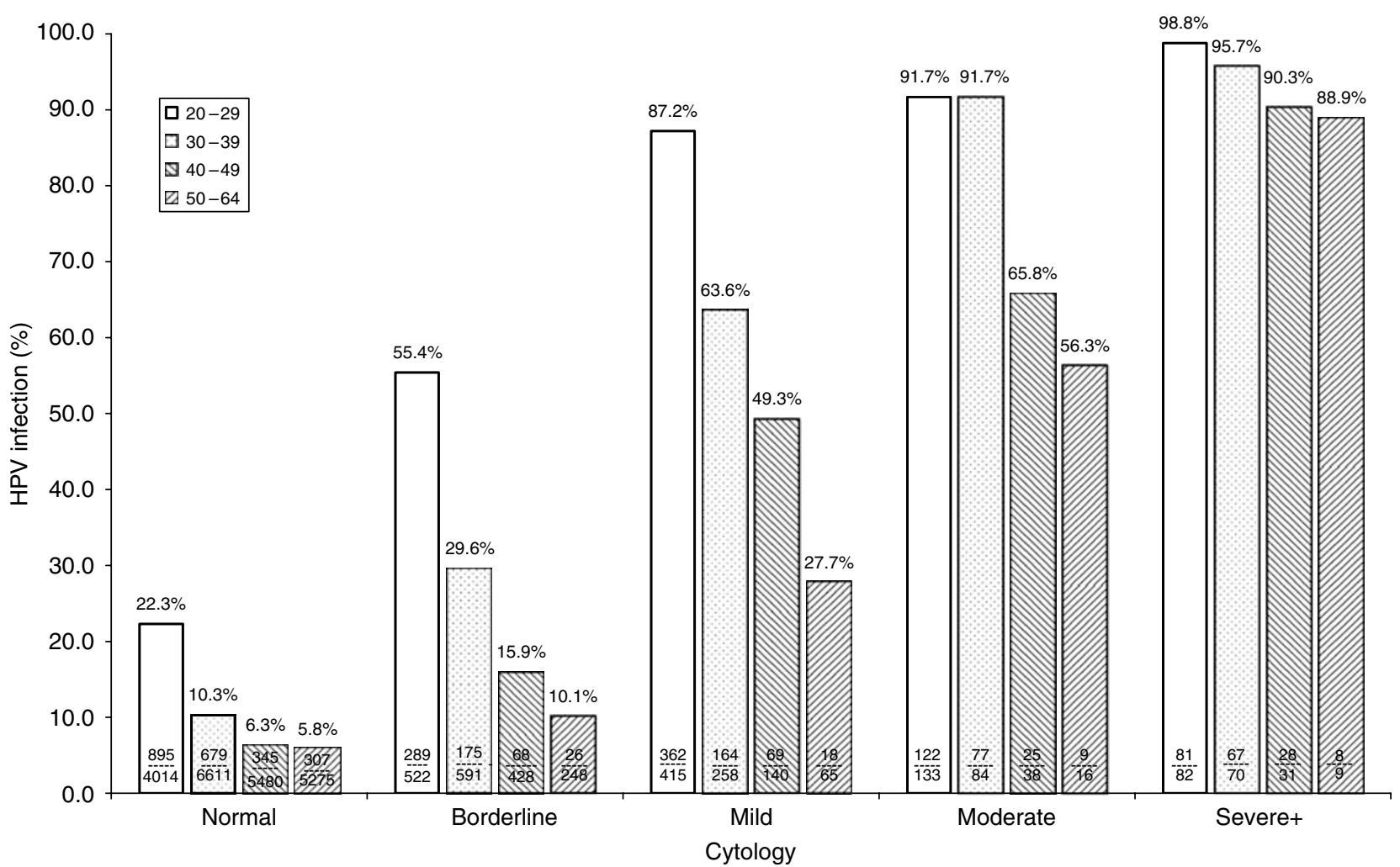

Figure 3 Prevalence of high-risk HPV (HR HPV) by Hybrid Capture 2 (HC2) by cytology grade within different age bands.

(mild), 92\% (moderate) and 99\% (severe). By age 50-64 years these rates had fallen to $10 \%$ (borderline), $28 \%$ (mild), $56 \%$ (moderate) and 89\% (severe).

Table 2 shows the prevalence of abnormal cytology by age and HPV detection. The overall proportion of women with high-grade (moderate or severe) cytology declined from $4.2 \%(215 / 5166)$ at age $20-29$ to $1.6 \%(223 / 13,731)$ at age $30-49$. This marked reduction in high-grade disease appears to be due entirely to the much lower overall HPV prevalence in women aged $30-49$ years, as the prevalence of high-grade abnormality in HPV-positive women was identical at age $20-29$ years (11.6\%: 203/1749) and at age 30-49 (11.6\%: 197/1697). Above age 50, however, high-grade cytological abnormality was diagnosed in only $4.6 \%(17 / 367)$ of HPV-positive women and in $0.4 \%$ overall. The number of women who would have been referred for follow-up if primary screening with HPV testing replaced cytology can also be seen in Table 2 . The proportion of smears that were abnormal was $22 \%(1153 / 5166)$ at aged $20-29$ years, $12 \%(1640 / 13731)$ at age $30-49$ years and $6.0 \%$ $(338 / 5613)$ at age $50-64$ years. The proportions HPV positive were $34 \%$ at $20-29$ years, $12 \%$ at $30-49$ years and $6.5 \%$ at $50-64$ years. 
Table 2 Cytological abnormality by age and high-risk HPV detection

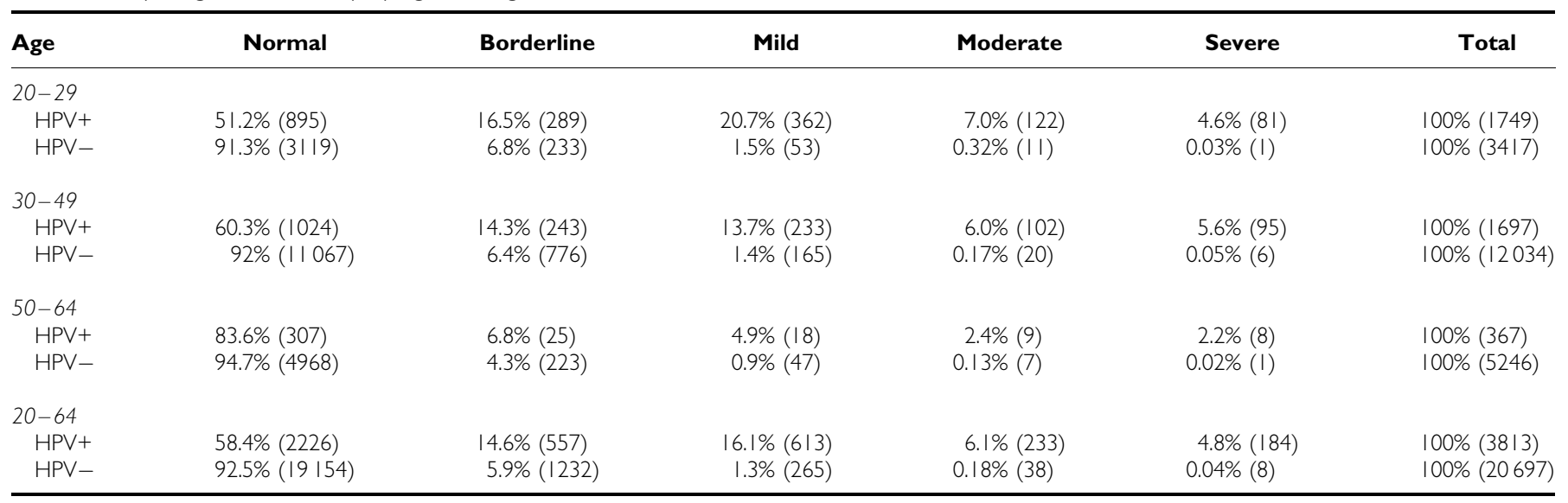

(): number of women HPV positive (negative) in that category.

Table 3 Prevalence of HPV 16, HPV I8 and other high risk HPV types by age, cytology and histology at entry

\begin{tabular}{|c|c|c|c|c|c|}
\hline Age & HC-II negatives & HPV 16 & HPV I8 (not HPV 16) & Other HC-II positives ${ }^{a}$ & Total \\
\hline $25-34$ & $4867(77.6 \%)$ & $320(5.1 \%)$ & $127(2.0 \%)$ & $957(15.3 \%)$ & $6271(100 \%)$ \\
\hline $55-64$ & 3037 (94.0\%) & $21(0.7 \%)$ & $7(0.2 \%)$ & $166(5.1 \%)$ & $3231(100 \%)$ \\
\hline \multicolumn{6}{|l|}{ Cytology } \\
\hline Mild & $265(30.2 \%)$ & $152(17.3 \%)$ & $40(4.6 \%)$ & $421(47.9 \%)$ & $878(100 \%)$ \\
\hline Moderate & 38 (14.0\%) & 107 (39.5\%) & $18(6.6 \%)$ & $108(39.9 \%)$ & $271(100 \%)$ \\
\hline Severe or worse & $8(4.2 \%)$ & $101(52.6 \%)$ & $17(8.8 \%)$ & $66(34.4 \%)$ & $192(100 \%)$ \\
\hline \multicolumn{6}{|l|}{ Histology } \\
\hline CINI or less & 318 (40.8\%) & 104 (13.3\%) & 48 (6.2\%) & 310 (39.7\%) & $780(100 \%)$ \\
\hline $\mathrm{CIN} 2$ & $15(7.1 \%)$ & 85 (40.1\%) & $15(7.1 \%)$ & 97 (45.7\%) & $212(100 \%)$ \\
\hline Total & 20697 (84.4\%) & $803(3.3 \%)$ & $272(1.1 \%)$ & $2738(11.2 \%)$ & $24510(100 \%)$ \\
\hline
\end{tabular}

SCC: squamous cell carcinoma. CGIN: cervical glandular intraepithelial neoplasia. ADCC: adenocarcinoma. ${ }^{2}$ Not HPV 16 or HPV I8. *** Women with abnormal cytology at entry but no histology (abnormal cytology resolved or still being followed-up cytologically).

Table 3 shows the proportions of women who tested positive for HPV 16, positive for HPV 18 (but not 16) and positive for hc2 in the absence of HPV 16 or HPV 18, in relation to age, cytology and histology at entry. The overall prevalence was 3.3\% for HPV 16 and $1.1 \%$ for HPV 18. Among women with any high-risk type detected by hc2, the proportion who had HPV 16 was $31 \%$ at age $20-24$ years, $23 \%$ at $25-34$ years and $12 \%$ at $35-64$ years. As the grade of cytological abnormality increased the proportion of women with HPV 16 infections increased from $1.5 \%$ in women with normal cytology to over $50 \%$ in those with severe dyskaryosis.

The lower part of Table 3 shows histological findings (worst histology within 2 years) among 1061 women with abnormal cytology at entry for whom a biopsy was obtained. No histology is yet available for the remaining 2069 women with an abnormal entry smear (bottom line, Table 3: abnormal cytology resolved or still being followed-up cytologically). Among women with histology, CIN3, CGIN or cancer was diagnosed in $46 \%$, (150/325) of women with HPV 16, 28\% (20/72) of those with HPV 18 (not 16), $20 \%(81 / 411)$ of those with other HPVs, and in only $3 \%$
$(8 / 253)$ of those whose entry smears were hc2 negative. The hc2 test was positive in $92 \%$ of women with CIN2 and $97 \%$ with CIN3, CGIN or cancer. HPV 16 was detected in $59 \%$ of women with CIN3 or squamous cell carcinoma. HPV 18 was detected in $38 \%$ of women with CGIN or adenocarcinoma but in only $6 \%$ with CIN3 or squamous cell carcinoma.

\section{DISCUSSION}

The ARTISTIC trial cohort represents the largest population of women in the UK to have undergone routine cervical screening with both LBC and HPV testing. The study population spanned the 20-64 age range of screened women when the trial opened, although the lower age threshold for routine cervical screening in England has since been increased from 20 to 25 years.

Our age-specific HPV positive rates in different grades of cytological abnormality were similar to those in the HART study in which over 10000 women were screened with conventional 
cytology and hc2 testing, but our overall HPV prevalence was slightly higher at each age. HPV prevalence in the HART study declined from $14.5 \%$ in women aged $30-34$ years to $3.8 \%$ in women aged 55-59 years (Cuzick et al, 2003); the corresponding rates in our cohort were $18.5 \%$ at $30-34$ years and $6 \%$ at $55-59$ years. Our higher rates may be partly due to regional differences in the UK (2004). The HART study was conducted in five centres across Britain, and the highest HPV prevalence was found in the Manchester area, where $16 \%$ of $30-34$ year olds were positive for HPV (P Sasieni, personal communication). There may also have been a continuing increase in HPV prevalence in this population. A study conducted in the same area as ARTISTIC between 1988 and 1993 reported HPV prevalence based on MY0911 consensus primer PCR of $18 \%$ in women aged $20-24$ years declining to $3 \%$ in women aged 50-54 years (Peto et al, 2004). Differences in HPV detection sensitivity may account for part of the disparity, but this doubling of prevalence within 12 years suggests a continuing epidemic rise in HPV prevalence.

Several useful conclusions relevant to the potential role for HPV testing in primary routine screening are suggested by the relationships between age, HPV detection and severity of cytology shown. In women with detectable HPV the prevalence of moderate dyskaryosis is 20 - to 30 -fold higher than in HPV negative women at all ages, and severe dyskaryosis is increased more than 100 -fold. The prevalence of mild dyskaryosis in HPV-positive women is about 10-fold higher than in HPV negative women below age 50 years and more than five-fold higher above age 50 years. Although a great majority $(87 \%)$ of women aged under 30 years with mild dyskaryosis are HPV positive, this proportion falls to 58\% (233/ $398)$ at age $30-49$ years and to only $28 \%(18 / 65)$ at age $50-64$ years suggesting a role for HPV triage. The prevalence of borderline abnormalities in HPV positive women is about twice as high as in HPV-negative women at each age, and although there may be some overcalling by $\mathrm{LBC}$, our results indicate that many borderline abnormalities are not caused by HPV. The prevalence of moderate or severe dyskaryosis in HPV-positive women is $11.6 \%$ throughout the premenopausal years suggesting that the natural history of HPV infection may be much the same in premenopausal women irrespective of age, although CIN3 is rarer

\section{REFERENCES}

Clifford GM, Gallus S, Herrero R, Munoz N, Snijders PJ, Vaccarella S, Anh PT, Ferreccio C, Hieu NT, Matos E, Molano M, Rajkumar R, Ronco G, de Sanjose S, Shin HR, Sukvirach S, Thomas JO, Tunsakul S, Meijer CJ, Franceschi S (2005) Worldwide distribution of human papillomavirus types in cytologically normal women in the International Agency for Research on Cancer HPV prevalence surveys: a pooled analysis. Lancet 366: $991-998$

Cuzick J, Szarewski A, Cubie H, Hulman G, Kitchener H, Luesley D, McGoogan E, Menon U, Terry G, Edwards R, Brooks C, Desai M, Gie C, Ho L, Jacobs I, Pickles C, Sasieni P (2003) Management of women who test positive for high-risk types of human papillomavirus: the HART study. Lancet 362: $1871-1876$

Harper DM, Franco EL, Wheeler CM, Moscicki AB, Romanowski B, RoteliMartins CM, Jenkins D, Schuind A, Costa Clemens SA, Dubin G (2006) Sustained efficacy up to 4.5 years of a bivalent L1 virus-like particle vaccine against human papillomavirus types 16 and 18: follow-up from a randomised control trial. Lancet 367: 1247-1255

\section{Appendix}

ARTISTIC Trial Study Group: HC Kitchener, Principal Investigator P Wheeler, Trial Co-ordinator; Epidemiology/Statistics: J Peto, M Almonte, C Gilham, C Roberts, S Moss; Cytopathology: in HPV-infected women aged 50 years or over. In women aged 30 years or over, our data suggest that the main effect of replacing cytology by HPV testing in primary screening would be the replacement of HPV negative abnormal smears, most of which would be borderline, by a similar number of HPV positive normal smears among women referred for follow-up. For those aged 20-29 years, however, the number who were HR HPV positive was $52 \%$ greater than the number with abnormal cytology, suggesting the need for a secondary test prior to colposcopy.

Primary screening with HPV testing in combination with cytology triage has been recommended only in women aged over 30 years (Sasieni and Cuzick, 2002; Cuzick et al, 2003), as HPV is so common in younger women. This conclusion seems questionable in the light of our results as high-grade dysplasia is as common among HPV-infected women aged under 30 years as in those age 30-49 years, and much commoner than in women aged over 50 years.

The ARTISTIC trial has also provided the largest collection of HPV typed primary screening cervical samples from the UK. The HPV type may be clinically important as the proportion of hc2 positive women who were infected with HPV16 increased with cytological abnormality, from $14 \%$ in those with normal cytology to $55 \%$ in those with severe dyskaryosis (Table 3). The HPV type might be used to determine whether to refer for colposcopy immediately, repeat the test, or defer any investigation until the next routine screen 3 years later. With the prospect of type 16/18 specific HPV prophylactic vaccines becoming available, data on these types in the screened population is of considerable importance in terms of what proportion of current abnormalities may still occur, notwithstanding a degree of cross protection reported for HPV 45 (Harper et al, 2006).

Women are still undergoing repeat cytology and colposcopy as necessary. It is therefore not yet possible to compare the sensitivity and specificity of cytology and HPV testing for detecting CIN2 and CIN3 at entry to the trial, particularly in relation to specific HPV types. As with any quantitative test, the performance of hc2 will depend on the choice of cutoff.

The second round of screening at 3 years began in July 2004 and we anticipate reporting final results at the end of 2007.

IARC (1995) Monographs on the Evaluation of Carcinogenic Risks to Humans No. 64. Human Papillomaviruses. IARC: Lyon

IARC (2004) Cervix Cancer Screening/IARC Working Group on the Evaluation of Cancer - Preventive Strategies. IARC: Lyon. IARC Handbook of Cancer Prevention

Parkin DM (2004) International variation. Oncogene 23: 6329-6340

Peto J, Gilham C, Deacon J, Taylor C, Evans C, Binns W, Haywood M, Elanko N, Coleman D, Yule R, Desai M (2004) Cervical HPV infection and neoplasia in a large population-based prospective study: the Manchester cohort. Br J Cancer 91: 942-953

Sasieni P, Cuzick J (2002) Could HPV testing become the sole primary cervical screening test? J Med Screen 9: 49-51

StataCorp (2004) Stata Statistical Software, Release 8.1. Stata Corporation Walboomers JM, Jacobs MV, Manos MM, Bosch FX, Kummer JA, Shah KV, Snijders PJ, Peto J, Meijer CJ, Munoz N (1999) Human papillomavirus is a necessary cause of invasive cervical cancer worldwide. J Pathol 189: $12-19$

M Desai, J Mather; Health Economics: R Dowie, B Stoykova; Virology Studies: A Bailey, A Sargent, A Turner, G Corbitt (deceased). 